\title{
Ghost excitonic insulator transition in layered graphite
}

\author{
D. V. Khveshchenko \\ Department of Physics and Astronomy, University of North Carolina, Chapel Hill, NC 27599
}

\begin{abstract}
Some unusual properties of layered graphite, including a linear energy dependence of the quasiparticle damping and weak ferromagnetism at low doping, are explained as a result of the proximity of a single graphene sheet to the excitonic insulator phase which can be further stabilized in a doped system of many layers stacked in the staggered $(A B A B \ldots)$ configuration.
\end{abstract}

The continuing interest in carbon-based materials has intensified both experimental and theoretical efforts to understand their electronic properties and interactiondriven transitions in these systems.

The problem of electronic instabilities in a single sheet of graphite has been previously studied by means of the Hartree-Fock and density functional methods with a focus on the short-ranged (including on-site and nearest neighbor) Hubbard-like repulsive interactions, and the inter-site repulsion was found to favor a charge density wave (CDW) ground state [1]. This analysis did not provide, however, a proper account of the Coulomb forces that remain long-ranged due to the lack of conventional screening in semimetals, such as graphite.

In contrast, the authors of Ref. [2] specifically focused on the role of the Coulomb interactions. From the renormalization group calculation in the second order in the dimensionless Coulomb coupling $g=2 \pi e^{2} / \varepsilon_{0} v$ they concluded that the renormalized coupling monotonously decreases at low energies and, therefore, it can not cause any instability of the gapless paramagnetic ground state of graphite.

Besides, the authors of Ref. [2] suggested a possible explanation for the experimentally observed [3] linear energy dependence of the quasiparticle damping (defined as the imaginary part of the quasiparticle dispersion $\left.\epsilon=E_{\mathbf{p}}+i \Sigma(\epsilon)\right)$ which they found to behave as $\Sigma(\epsilon) \sim \epsilon / \ln ^{2} \epsilon$ at low energies. However, the large estimated value of the bare coupling constant $(g \gtrsim 10)$ calls these results into question and warrants further investigation.

In the present Letter, we revisit the problem of the Coulomb interacting electrons in layered graphite and study the nature of the ground state and the quasiparticle spectrum at strong coupling. This time around, we employ a non-perturbative approach by solving a nonlinear equation for the electron Green function, which will allow us to ascertain the status of the previous results obtained in perturbation theory and test our theoretical predictions against several pieces of the existing experimental evidence.

The semimetallic energy band structure of a single graphene sheet gives rise to the conduction and valence bands' touching each other in the two inequivalent $K$-points located at the corners of the hexagonal twodimensional (2D) Brillouin zone. In the absence of in- teractions, the low-energy quasiparticle excitations with the momenta in the vicinity of these points labeled as $i=1,2$ have linear dispersion $E_{\mathbf{p}}^{(0)}= \pm v \mathbf{p}$, the velocity $v$ being proportional to the width of the electronic $\pi$-band $t \approx 2.4 \mathrm{eV}$ [4].

These excitations can be formally described by a pair of two-component (Weyl) spinors $\psi_{i \sigma}$, each carrying a spin index $\sigma$, which are composed of the Bloch states residing on the two different sublattices of the bi-partite hexagonal lattice of the graphene sheet. In what follows, we choose to combine them into one four-component Dirac spinor $\Psi_{\sigma}=\left(\psi_{1 \sigma}, \psi_{2 \sigma}\right)$ and also treat the number of the spin components $N$ as an adjustable parameter, the physical case corresponding to $N=2$.

The use of the Dirac spinor representation allows one to cast the free quasiparticle Hamiltonian in the relativistic-like form where $v$ is playing the role of the speed of light

$$
H_{0}=i v \sum_{\sigma=1}^{N} \int_{\mathbf{r}} \bar{\Psi}_{\sigma}\left(\hat{\gamma}_{1} \nabla_{x}+\hat{\gamma}_{2} \nabla_{y}\right) \Psi_{\sigma}
$$

where $\bar{\Psi}_{\sigma}=\Psi_{\sigma}^{\dagger} \hat{\gamma}_{0}$ and the reducible representation of the $4 \times 4 \gamma$-matrices $\hat{\gamma}_{0,1,2}=\left(\tau_{3}, i \tau_{2},-i \tau_{1}\right) \otimes \tau_{3}$ given in terms of the triplet of the Pauli matrices $\tau_{i}$ satisfies the usual anticommutation relations: $\left\{\hat{\gamma}_{\mu}, \hat{\gamma}_{\nu}\right\}=$ $2 \operatorname{diag}(1,-1,-1) \mathbf{1} \otimes \mathbf{1}$.

In the four-spinor representation, the electron Coulomb interaction reads as

$$
H_{C}=\frac{v}{4 \pi} \sum_{\sigma, \sigma^{\prime}=1}^{N} \int_{\mathbf{r}, \mathbf{r}^{\prime}} \bar{\Psi}_{\sigma}(\mathbf{r}) \hat{\gamma}_{0} \Psi_{\sigma}(\mathbf{r}) \frac{g}{\left|\mathbf{r}-\mathbf{r}^{\prime}\right|} \bar{\Psi}_{\sigma^{\prime}}\left(\mathbf{r}^{\prime}\right) \hat{\gamma}_{0} \Psi_{\sigma^{\prime}}\left(\mathbf{r}^{\prime}\right)
$$

Despite the apparent lack of the Lorentz invariance, Eq.(2) remains invariant under arbitrary $U(2 N)$ rotations of the $2 N$-component vector $\left(\Psi_{L \sigma}, \Psi_{R \sigma}\right)$ composed of the chiral Dirac fermions defined as: $\Psi_{L, R \sigma}=\frac{1}{2}(\mathbf{1} \pm$ $\left.\hat{\gamma}_{5}\right) \Psi_{\sigma}$, where the matrix $\hat{\gamma}_{5}=\mathbf{1} \otimes \tau_{2}$ anticommutes with any $\hat{\gamma}_{\mu}$.

The chiral invariance of Eqs. $(1,2)$ brings about the possibility of spontaneous chiral symmetry breaking (CSB), akin the phenomenon that has long been studied in the relativistic fermion theories. The CSB transition manifests itself in the appearance of a fermion mass and gapping of the fermion spectrum, thus breaking 
the continuous chiral symmetry from $U(2 N)$ down to $U(N) \otimes U(N)$ and developing a non-zero expectation value $<\sum_{\sigma}^{N} \bar{\Psi}_{\sigma}(\mathbf{r}) \Psi_{\sigma}(\mathbf{r}) \quad>=<\sum_{\sigma}^{N}\left(\psi_{\sigma}^{\dagger}(A) \psi_{\sigma}(A)-\right.$ $\left.\psi_{\sigma}^{\dagger}(B) \psi_{\sigma}(B)\right)>$. The latter corresponds to the electron density modulation which alternates between the two sublattices ( $A$ and $B$ ).

In light of the above, one can identify the CSB order parameter with the site-centered CDW and thus relate it to the $p=0$ value of the gap function $\Delta_{p}$ appearing in the renormalized (and, generally, non-Lorentz invariant) fermion Green function

$$
\hat{G}_{p}=Z_{p}\left[\left(\epsilon \hat{\gamma}_{0}-v_{p} \vec{p} \hat{\vec{\gamma}}\right)+\Delta_{p}\right]^{-1}
$$

where the interaction effects can also give rise to the nontrivial wave function $\left(Z_{p}\right)$ and velocity $\left(v_{p} / v\right)$ renormalization factors.

Because of its intrinsically non-perturbative nature, the phenomenon of CSB evades weak-coupling analysis based on perturbation theory. Nonetheless, similar to its relativistic counterpart [5], the CSB can be revealed by a non-perturbative solution of the system of nonlinear equations for the fermion Green function (hereafter $\left.\hat{p}=\epsilon \hat{\gamma}_{0}-v \vec{p} \hat{\vec{\gamma}}\right)$

$$
\hat{G}_{p}^{-1}=\hat{p}+\int \frac{d^{3} k}{(2 \pi)^{3}} \Gamma_{p, k} \hat{\gamma}_{0} \hat{G}_{p+k} \hat{\gamma}_{0} V_{k},
$$

vertex function $\Gamma_{p, k}$, and effective Coulomb interaction $V_{k}=1 /[(\mathbf{q} / g v)+N \chi(\omega, \mathbf{q})]$ which gets strongly modified by the intra-layer polarization of the Dirac fermions

$$
\chi_{k}=\operatorname{Tr} \int \frac{d^{3} p}{(2 \pi)^{3}} \Gamma_{p, k} \hat{\gamma}_{0} \hat{G}_{p+k} \hat{\gamma}_{0} \hat{G}_{p}
$$

A further analytical progress is hindered by the fact that, as a result of the interaction's $V_{k}$ being explicitly nonLorentz invariant, the gap function $\Delta_{p}$ can feature separate dependencies on the energy $\epsilon$ and momentum $\mathbf{p}$ variables. Therefore, we choose to proceed directly with the finite temperature counterpart of Eq.(4), in which case the Lorentz invariance is broken regardless of the symmetry of the fermion interactions.

In order to get a preliminary insight into the problem we resort to the same approximations as those made in the previous studies of CSB in the context of $Q E D_{3}$ [5]. To this end, we first neglect the wave function, velocity, and vertex renormalizations $\left(Z_{p}=v_{p} / v=\Gamma_{p, k}=1\right)$ in Eq.(4) whose scalar part then becomes a closed equation for the fermion gap function. As shown in [5], neglecting the above renormalizations in the gap equation suffices for establishing the existence of its non-trivial solution(s) and estimating a critical value $N_{c}$ of the only remaining free parameter, the number of fermion species.

Taking the sum over the discrete Matsubara frequencies we then arrive at the momentum-dependent gap equation
$\Delta_{\mathbf{p}}=\int \frac{d^{2} \mathbf{k}}{8 \pi^{2}} \frac{\tanh E_{\mathbf{k}} / 2 T}{E_{\mathbf{k}}} \frac{\Delta_{\mathbf{k}}}{|\mathbf{k}-\mathbf{p}| / g v+N \chi(0, \mathbf{k}-\mathbf{p})}$

where $E_{\mathbf{p}}=\sqrt{v^{2} \mathbf{p}^{2}+\Delta_{\mathbf{p}}^{2}}$. Next, we approximate the exact finite temperature fermion polarization (5) by that computed in the massless case. By doing so, we overestimate the contribution of the fermion momenta $\mathbf{p} \lesssim \Delta_{\mathbf{p}}$ which is, however, unimportant, as long as the gap remains much smaller than the high-momentum cutoff $\Lambda$ comparable to the maximum span of the Brillouin zone. As shown below, this condition is indeed satisfied for $N$ close to the critical value $N_{c}$.

At $\Delta_{\mathbf{p}}=0$ the fermion polarization is given by the approximate formula

$$
\begin{array}{r}
\chi(0, \mathbf{q})=\frac{2 T}{\pi v_{F}^{2}} \int_{0}^{\infty} d x \ln \left[2 \cosh \left(\frac{v \mathbf{q}}{2 T} \sqrt{x(1-x)}\right)\right] \\
\approx \frac{1}{8 v^{2}}\left[v \mathbf{q}+c T \exp \left(-\frac{v \mathbf{q}}{c T}\right)\right]
\end{array}
$$

which, for $c=16 \ln 2 / \pi$, provides an up to a few percent accurate interpolation between the two opposite limits: $v \mathbf{q} \gg T$ where Eq.(团) agrees with the zero-temperature result $\chi_{k} \propto \sqrt{k^{2}}$ and $v \mathbf{q} \ll T$ where it exhibits thermal screening $\chi_{0} \propto T$ [6].

Notably, at strong coupling $(g \gg 1)$ the screened Coulomb interaction $V_{k}$ becomes independent of the bare coupling constant and assumes a universal form $V_{k} \approx$ $1 / \chi(0, \mathbf{k})$ governed by the fermion polarization $(7)$.

Upon differentiating Eq.(6) with respect to the momentum $\mathbf{p}$ one finds that for $E_{\mathbf{p}}>T$ this non-linear integral equation reduces to a linear differential one

$$
\frac{d^{2} \Delta_{\mathbf{p}}}{d \mathbf{p}^{2}}+\frac{2}{\mathbf{p}} \frac{d \Delta_{\mathbf{p}}}{d \mathbf{p}}+\frac{2}{\pi N} \frac{\Delta_{\mathbf{p}}}{\mathbf{p}^{2}}=0
$$

which has to be supplemented by the boundary conditions $\Delta_{0}<\infty$ and $\left.\left(\Delta_{\mathbf{p}}+\mathbf{p} d \Delta_{\mathbf{p}} / d \mathbf{p}\right)\right|_{\mathbf{p}=\Lambda}=0$.

In turn, Eq.(8) can be readily identified with the radial Schroedinger equation for the $s$-wave zero energy level in the potential that behaves as $\propto 1 / \mathbf{p}^{2}$ for $\mathbf{p}>T / v$. From the textbook solution of this problem [7] we infer that for $N>N_{c}=8 / \pi$ there are two independent solutions $\Delta_{\mathbf{p}}^{ \pm} \propto 1 / \mathbf{p}^{\left(1 \pm \sqrt{1-N_{c} / N}\right) / 2}$, neither of which can satisfy the above boundary conditions. In contrast, for $N<N_{c}$ there exists a solution with infinitely many nodes, consistent with the infinite number of the negative energy levels in the $1 / \mathbf{p}^{2}$-potential. Thus, only in this "centerward downfall" regime does the solution

$$
\Delta_{\mathbf{p}} \sim \frac{T^{3 / 2}}{\sqrt{v \mathbf{p}}} \sin \left(\frac{1}{2} \sqrt{\frac{N_{c}}{N}-1} \ln \frac{v \mathbf{p}}{T}\right)
$$

monotoneously decrease in the interval $T / v<\mathbf{p}<\Lambda$ and satisfy the boundary condition at $\mathbf{p}=\Lambda$ which reads as 


$$
\sqrt{\frac{N_{c}}{N}-1} \ln \frac{v \Lambda}{T}=2 \pi n-2 \tan ^{-1}\left(\frac{1}{2} \sqrt{\frac{N_{c}}{N}-1}\right)
$$

where $n$ is a positive integer. We note, in passing, that due to the formal similarity between the underlying equations, a solution with the similar critical properties has also been discovered in the context of the 2D Cooper pairing near the antiferromagnetic instability [8].

The highest critical temperature $T_{c}(N)$ below which the CSB order parameter sets in corresponds to $n=1$ :

$$
T_{c}(N) \approx v \Lambda \exp \left(-\frac{2 \pi}{\sqrt{N_{c} / N-1}}\right)
$$

According to Eq.(11), at non-zero temperatures the critical number of fermion species gets reduced: $N_{c}(0)-$ $N_{c}(T) \approx 4 \pi^{2} N_{c}(0) / \ln ^{2}(v \Lambda / T)$.

In the case of $Q E D_{3}$, the progressively more and more refined numerical simulations [6] have demonstrated that the results of the original analytical approach of Ref. [5] which yielded a solution similar to Eq.(9) remain robust against relaxing the above approximations and taking into account both the wave function renormalization and the vertex function satisfying the Ward identity $\Gamma_{p, 0}=Z_{p}$.

Likewise, a numerical analysis of the coupled Eqs.(4) and (5) confirms the existence of the solution (9) in a whole domain bordered by the critical line (11) in the $N-T$ plane [9].

Also, the numerically evaluated characteristic ratio $2 \Delta_{0} / T_{c} \approx 10$ appears to be close to that found in Ref. [6] which is substantially greater than the BCS value corresponding to the solution $\Delta_{\mathbf{p}}=$ const of the gap equation with a momentum-independent kernel.

As far as the nature of the CSB transition is concerned, the observed $N$-dependence of the zero-momentum fermion gap $\Delta_{0} \propto \exp \left(-2 \pi / \sqrt{N_{c} / N-1}\right)$ prompts one to identify the breaking of the continuous chiral symmetry as a topological (Kosterlitz-Thouless-type) phase transition in $2+1$ dimensions [5. Thus, the finite temperature CSB transition occurs between the two phases which are both chirally symmetrical, and therefore a bosonic Goldstone mode must be present in the quasi-ordered phase.

As regards the critical number of fermion species itself, the recent symmetry-based argument made in the context of $Q E D_{3}$ shows that the gap equation systematically overestimates the actual value of $N_{c}$ which may, in fact, be as low as $3 / 2$ [10 while the gap equation yields $N_{c}^{Q E D}=32 / \pi^{2}[$ [5].

The demonstrated formal relationship between the finite temperature $Q E D_{3}$ and the problem of a single graphene sheet suggests that in the latter case the actual critical number of fermion species might also be less than two, hence no CSB occurs.

Nonetheless, even at $N>N_{c}$ the nearby CSB transition, albeit unreachable at any $g$, can still have a profound effect on the quasiparticle spectrum both above and below the crossover into the quantum-critical regime associated with the zero-temperature quantum-critical point at $N_{c}$.

In the quantum disordered (low-temperature) regime $T \lesssim T^{\star}(N)$, where the crossover temperature $T^{\star}(N)$ vanishes at $N \rightarrow N_{c}+0$ in the same manner as $T_{c}(N)$ given by Eq.(11) for $N \rightarrow N_{c}-0$, the only solution of Eqs.(4) and (5) is a massless fermion propagator which exhibits a suppression of the residue of the bare quasiparticle pole: $Z_{p} \rightarrow 0$ for $p \rightarrow 0$, while the velocity $v_{p}$ undergoes singular renormalization and monotonously increases with decreasing momentum, unlike in the Lorentinvariant $Q E D_{3}$ where it remains constant.

By contrast, in the quantum-critical regime $T \gtrsim$ $T^{\star}(N)$ the fermion propagator features a simple pole, whereas the temperature- (but no longer momentum-) dependent factors $Z_{T}$ and $v_{T}$ control its residue and the effective velocity, respectively. The fermion damping is then determined by the self-consistent equation

$$
\begin{array}{r}
\Sigma(\epsilon)=\frac{1}{N} \int \frac{d \omega d \mathbf{q}}{(2 \pi)^{3}}\left\{\tanh \frac{\varepsilon+\omega}{2 T}-\operatorname{coth} \frac{\omega}{2 T}\right\} \times \\
\operatorname{Im}\left[\frac{\varepsilon+\omega+i \Sigma(\varepsilon+\omega)}{(\varepsilon+\omega+i \Sigma(\varepsilon+\omega))^{2}-v^{2} \mathbf{q}^{2}}\right] \operatorname{Im} \frac{1}{\chi(\omega, \mathbf{q})}
\end{array}
$$

which yields the universal solution: $\Sigma(\epsilon) \sim \max (\epsilon, T)$, in a general agreement with the time-resolved two-photon photoemission data taken in the energy range $0.4<\epsilon<$ $2 \mathrm{eV}[3]$. One can expect that this characteristic signature of the CSB-related quantum-critical behavior will be even more pronounced in the case of a graphite monolayer deposited on an insulating surface, whereas a conducting substrate would hamper the possibility of observing the linear damping due to strong metallic screening.

The predicted linear damping should be possible to observe in angular-resolved photoemission which can specifically probe the vicinity of the $K$-points. On the contrary, the angular-averaged data are going to be affected by such details of the graphite bandstructure as, e.g., the saddle point in the quasiparticle dispersion which occurs at $\epsilon \approx 1.5 \mathrm{eV}$ if the momentum resides at one of the $M$-points of the Brillouin zone. This saddle point was recently argued to be a likely cause of the additional plateau-like feature observed in the angularaveraged $\Sigma(\epsilon)$ [11 which is, therefore, unrelated to the many-body phenomena discussed in this Letter.

In a stack of graphite layers with the inter-layer spacing $d$, the screened intra-layer Coulomb interaction remains dominated by the polarization $\chi_{k}$ only at $\mathbf{q}>1 / N g d$. At still lower momenta the kernel in Eq.(6) becomes less singular $\left(V_{k} \propto 1 / \sqrt{\mathbf{q}}\right)$, thus reducing the range of temperatures and/or energies where the quantum-critical behavior associated with the nearby CSB transition can be observed in electron photoemission.

A finite inter-layer hopping $t_{\perp} \approx 0.27 \mathrm{eV}$ provides another cutoff below which the particle-hole pairing cor- 
relations cease to drive the system towards the opening of the excitonic gap, and the quasiparticle damping becomes quadratic in energy for $\epsilon \lesssim t_{\perp}$.

In contrast, the inter-layer Coulomb interaction has the opposite effect of nudging a stack of graphite layeres closer to the CSB instability. To elucidate this point, we recall that the common form of graphite has a crystal structure of well-separated hexagonal layers stacked in a staggered $(A B A B \ldots)$ configuration. As a result, each layer gets naturally divided into two sublattices formed by the atoms positioned just above and below the centers and corners of the hexagons in the two adjacent layers, respectively.

Thus the inter-layer Coulomb repulsion strengthens the system's propensity towards developing the CDW instability by favoring spontaneous depletion of one of the two sublattices (accompanied by excess occupation of the complementary one) which alternates between the layers in order to keep the electrons in the neighboring layers as far apart as possible.

Formally, one can incorporate this effect of the interlayer Coulomb interaction into the effective single-layer description by adding a short-ranged four-fermion term $\lambda\left(\sum_{\sigma}^{N} \bar{\Psi}_{\sigma} \Psi_{\sigma}\right)^{2}$ into Eq.(2). With such a term present, the CSB transition occurs for any number of fermion species, including $N>N_{c}$, provided that the strength of this coupling exceeds a certain critical value $\left(\lambda>\lambda_{c}(N)\right)$ which grows with $N$ [9].

By further elaborating on the solution of Eq.(6) obtained in the physical case $N=2$ we also find out that, upon doping the system of graphite layers, the excitonic insulating ground state tends to spontaneously develop a non-zero spin polarization $<\psi_{\sigma}^{\dagger}(A) \psi_{\sigma}(A)+$ $\psi_{\sigma}^{\dagger}(B) \psi_{\sigma}(B)>\sim \mu \delta_{\sigma \sigma_{0}}$ proportional to the chemical potential $\mu$ introduced by doping [9].

This observation sheds light on the possible origin of weak ferromagnetism which was recently observed in highly oriented pyrolitic graphite (HOPG) [12]. Our fundings suggest that the latter might be not that different from the mechanism proposed in the recent studies of hexaborides believed to be 3D excitonic insulators [13]. Notably, the authors of Ref. [12] excluded magnetic impurities as a possible cause of the ferromagnetic behavior of the magnetization hysteresis loops (also consistent with the electron spin resonance data) observed in the samples showing the insulator-like temperature dependence of the resistivity. Elaborating on the analysis of Ref. [13] we predict that if the excitonic instability proved to be at work, the weak ferromagnetism would have to disappear above a certain level of doping corresponding to the chemical potential $\mu_{c} \sim \Delta_{0}$.

In summary, we study the problem of the Coulomb interaction-driven electronic instabilities in layered graphite and propose a new explanation for the experimentally observed linear quasiparticle damping which might be a result of the relative proximity of a single graphene sheet to the zero-temperature quantum-critical point corresponding to the transition to the $2 \mathrm{D}$ excitonic insulator. In lightly doped layered graphite, the excitonic instability give rise to the formation of the site-centered CDW ground state exhibiting weak ferromagnetism, as observed experimentally. Together with the recently proposed explanation [14] of the apparent semimetal-insulator transition in applied magnetic field [15] as a phenomenon of the magnetic field-driven CSB, it lends a further support to the discovered formal relationship between the problem of layered graphite and the behaviors found in the relativistic theories of the $2 \mathrm{D}$ Dirac fermions.

The author is grateful to Y. Kopelevich for communicating his experimental results prior to publication and S. Washburn for a discussion. This research was supported by the NSF under Grant No. DMR-0071362.

[1] A. L. Tchougreeff and R. Hoffmann, J. Phys. Chem.96, 8993 (1992); F. R. Wagner and M. B. Lepetit, ibid 100, 11050 (1996).

[2] J. Gonzalez, F. Guinea, and M. A. H. Vozmediano, Nucl. Phys. 424, 595 (1994); Phys. Rev. Lett. 77, 3589 (1996); Phys. Rev. B59, 2474 (1999).

[3] S. Xu et al, Phys. Rev. Lett. 76, 483 (1996); K. Ertel et al, Appl. Phys. B68, 439 (1999).

[4] G. Semenoff, Phys. Rev. Lett. 53, 2449 (1984); F. D. M. Haldane, ibid 61, 2015 (1988).

[5] T. Appelquist, D. Nash, and L. C. R. Wijewardhana, Phys. Rev. Lett. 60, 2575 (1988); T. Appelquist, J. Terning, and L. C. R. Wijewardhana, ibid 75, 2081 (1995).

[6] I. J. R. Aitchison et al, Phys. Lett. B294, 91 (1992); I. J. R. Aitchison and M. Klein-Kreisler, Phys. Rev. D50, 1068 (1994); G. Triantaphyllou, Phys. Rev. D58, 065006 (1998).

[7] L. D. Landau and E. M. Lifshitz, Quantum Mechanics (Pergamon, Oxford, 1981), Chapter 5.

[8] A. Abanov, A. V. Chubukov, and A. M. Finkelstein, Europhys. Lett. 54, 488 (2001); A. Abanov, A. V. Chubukov, and J. Schmalian, ibid 55, 369 (2001).

[9] D. V. Khveshchenko and H. Leal, unpublished.

[10] T. Appelquist, A. G. Cohen, and M. Schmaltz, Phys. Rev. D60, 045003 (1999).

[11] G. Moos et al, cond-mat/0106486; C. D. Spataru et al, cond-mat/0107043.

[12] Y. Kopelevich et al, J. Low Temp. Phys. 119, 691 (2000).

[13] M. E. Zhitomirsky, T. M. Rice, and V. I. Anisimov, Nature 402, 251 (1999); L. Balentz and C. M. Varma, Phys. Rev. Lett. 84, 1246 (2000); V. Barzykin and L. P. Gorkov, ibid 84, 2207 (2000).

[14] D. V. Khveshchenko, Phys. Rev. Lett. 87, 206401 (2001).

[15] H. Kempa et al, Solid State Commun. 115, 539 (2000); M. S. Sercheli et al, cond-mat/0106232. 